\title{
EL USO DE GPS PARA EL ANÁLISIS DEL COMPORTAMIENTO ESPACIO-TEMPORAL DE LOS TURISTAS. PRE-TEST EN EL VALLE DE BOÍ
}

\author{
José A. Donaire* \\ Núria Gali* \\ Universidad de Girona \\ Marcelo Royo-Vela** \\ Universidad de Valencia
}

\section{RESUMEN}

Este artículo es un análisis exploratorio sobre el uso de los GPS para estudiar el comportamiento de los visitantes en un espacio abierto. El Valle de Boí es un escenario turístico que ofrece diversos recursos de primer orden, por lo que el estudio de los recorridos de los visitantes permite analizar la respuesta a estímulos muy diferentes. Los resultados demuestran que el recurso del GPS en espacios abiertos permite ampliar la información sobre el uso social del espacio turístico y abre las puertas a nuevas formas de análisis.

Palabras clave: comportamiento turístico, GPS, actividad espacio-temporal, Valle de Boí.

Using GPS to analysis time-space tourists' behaviour. A pre-test study in Boí Valley

\section{ABSTRACT}

This article is an exploratory analysis of the use of GPS for the study of visitors' behaviour in an open space. The Boi Valley is an open space that offers important tourism resources, so

Recibido: 9 de abril de 2014

Devuelto para su revisión: 20 de junio de 2014

Aceptado: 28 de julio de 2014

* Facultad de Turismo. Universidad de Girona. Pl. Ferrater Mora,1. 17071 GIRONA (España). E-mail: ja.donaire@udg.edu,nuria.gali@udg.edu

** Facultad de Economía. Universidad de Valencia. Avda. De los Naranjos, s/. 46022 VALENCIA (España). E-mail: marcelo.royo@uv.es 
that the study of visitors' paths permits to analyse the response to many different stimulus. The results show that the use of GPS in open spaces give information of the social use of the tourism space and allows to use new forms of analysis.

Key words: tourist behaviour, GPS, time-space activity, Boí Valley.

\section{INTRODUCCIÓN}

El estudio del comportamiento turístico ha sido y es un tema de gran interés académico. Investigadores de disciplinas diversas se han aproximado al tema desde diferentes perspectivas. Por un lado, la psicología y la sociología se han interesado, sobre todo, en el comportamiento motivacional de los turistas; el marketing, por otro lado, en el proceso de toma de decisiones y el comportamiento de los turistas; la economía en el gasto de los turistas y la geografía en el movimiento físico de los visitantes.

Independientemente del área de estudio y su acercamiento al tema, está claro que el comportamiento turístico tiene lugar en un determinado espacio y en un determinado tiempo (Xiao-Ting y Bi-hu, 2012); y como apuntan Thornton et al. (1997) conocer el comportamiento espacial y temporal de los turistas permite mejorar la gestión de los atractivos, optimizar la administración de los servicios básicos, ampliar los flujos de visitantes o renovar estrategias de marketing, entre otras cosas. En definitiva, conocer el proceder de los visitantes en un destino permite mejorar la gestión turística del mismo.

El estudio del comportamiento espacio-temporal de los turistas se ha basado en diversos métodos de recopilación de datos, tales como la observación participada y no participada, los diarios de viajes, los mapas de comportamiento, el estudio de las fotografías de los turistas, o, también, el análisis de blogs de viajes.

Entre los métodos más usados están los diarios de viajes, donde el turista va relatando sus actividades en diarios más o menos estructurados (Pearce, 1988; Debbage, 1991; Thornton et al., 1997; Haldrup, 2004; Xiao-Ting y Bi-Hu, 2012). Los diarios de viaje se complementan, frecuentemente, con los mapas de comportamiento donde se dibujan los recorridos y los flujos de visitantes por el espacio (Pearce, 1988; Lew y Mckercher, 2006; Petterson y Zillinger, 2011; Xiao-Ting y Bi-Hu, 2012). Los blogs turísticos online, de viajeros por el mundo, son los actuales diarios de viajes. En ellos se pueden seguir los movimientos espaciales y temporales de sus autores y se pueden georreferenciar en un mapa (Molz, 2010). Lógicamente, los diarios de viaje y los mapas de comportamiento siempre dependen del entusiasmo y grado de implicación del sujeto.

La observación directa es una de las metodologías más adecuadas para el estudio del comportamiento efectivo, ya que permite disponer de una gran cantidad de información detallada sobre la interacción entre sujeto y objeto, es decir, entre los visitantes y el espacio visitado. Existen diferentes tipos de observación. Por un lado, algunos investigadores han apostado por la observación directa participada; es decir, el investigador forma parte del grupo que está observando. En esta línea destacan los estudios de Hartmann (1988) acompañando a pequeños grupos de turistas (2-5 componentes) durante un día de visita 
en Múnich; o Bowen (2002) que estudia un pequeño grupo de turistas, a los que acompaña a lo largo de un viaje organizado desde el Reino Unido a Malasia y Singapur. El principal problema de la observación participada es la influencia que el investigador ejerce en el visitante, además del tiempo que supone la recogida de información.

Por otro lado, la observación no participada implica que el investigador observa al visitante sin acompañarle. Normalmente se observa desde una distancia prudente y sin que el turista sea consciente de que está siendo observado. Uno de los primeros referentes en observación no participada es Hartmann (1988) en el estudio, antes mencionado, sobre el comportamiento de los jóvenes turistas canadienses y norteamericanos en Múnich, donde también se utilizó el método de la observación no participada. Murphy (1992) utiliza la técnica para el estudio del turismo urbano en la ciudad de Victoria en la Isla de Vancouver. Keul y Küheberger (1997) observan a los turistas en la ciudad de Salzburg durante periodos de 15 minutos y en diferentes franjas horarias, para el estudio del comportamiento espacio-temporal de los turista en la ciudad. Uno de los resultados más reveladores fue que los turistas que visitan el espacio por primera vez caminan mucho más rápido y paran mucho más tiempo, que los repetidores. Galí y Donaire (2006) también estudiaron el comportamiento de los visitantes en el Barrio Viejo de Girona, a partir de la observación no participada. El trabajo se basó en la teoría de grafos y en la modelización del espacio turístico en una suma de arcos y de nodos, por donde transitan los visitantes. Sin embargo, la observación no participada presenta el problema del elevado coste que suponen este tipo de investigaciones, y con una presencia muy activa del investigador en el trabajo de campo. Una posibilidad para reducir estos elevados costes es el uso de instrumentos de grabación, tales como la fotografía aérea, las cámaras de vídeo, etc. (Hartmann, 1988; Petterson y Zillinger, 2011).

Otros trabajos han realizado una aproximación muy diferente al estudio de la actividad espacio-temporal de los visitantes, a partir del análisis de las fotografías realizadas por los mismos turistas durante sus viajes. El método más antiguo y más ampliamente aceptado de acceso y análisis de imágenes turísticas ha sido el VEP (Visitor Employed Photography), es decir usar a los turistas como fotógrafos - empleados (Haywood, 1990; Mackay y Couldwell, 2004; Caton y Santos, 2008; Garrod, 2008). El VEP es una técnica en la que los turistas ofrecen a los investigadores las fotografías que han realizado a lo largo de un viaje. El problema nuevamente es que se requiere un elevado grado de implicación del turista en el trabajo de campo, lo que puede influir en el tamaño de la muestra, y que el contacto previo entre investigador y visitante puede condicionar las capturas fotográficas (Donaire y Galí, 2011). Otra técnica es el uso de fotografías que se han publicado en las redes sociales como fuente de información, por ejemplo el estudio de Donaire et al. (2014) analizando las fotografías de los turistas del Valle de Boí en Flickr o el trabajo realizado por Crandall et al. (2009), que analiza la «huella fotográfica» proporcionada por los visitantes a través de 35 millones de imágenes georreferenciadas.

En la actualidad, se está generalizando el uso de Sistemas de Posicionamiento Global (en adelante GPS) para el estudio de la actividad espacio-temporal de los visitantes en lugares turísticos. La recogida sistemática de información permite automatizar los registros y crear modelos de comportamiento, siguiendo el modelo clásico de Hägerstrand (Hägerstrand, 1970). De hecho, empieza a ser una práctica recurrente en el mundo académico y, 
últimamente, está apareciendo un elevado número de estudios que analizan los patrones de movimiento de los turistas usando GPS (Shoval y Isaacson, 2006, 2007, 2010; Chhetri y Arrowsmith, 2008; Tchetchick et al., 2009; Zillinger, 2010; Russo et al., 2010; Pettersson y Zillinger, 2011; Shoval et al., 2011; Mckercher et al., 2012; Orellana et al., 2012; Wolf et al., 2012; Hallo et al., 2012; Birenboim et al., 2013; Bauder, 2014; Grinberger et al., 2014), con el fin de recoger datos sobre su comportamiento espacial y temporal con resultados bastante acertados.

Los GPS puede rastrear la ubicación de un turista cada segundo con una precisión muy elevada. Por lo tanto, proporcionan una gran cantidad de información, con un nivel de detalle muy alto sobre el comportamiento de los peatones (Shoval y Isaacson, 2007). Por otra parte, los datos recogidos pueden ser fácilmente analizados utilizando Sistemas de Información Geográfica (GIS), que facilitan información muy precisa sobre los patrones de comportamiento turísticos, el tiempo total transcurrido, el tiempo en cada nodo, la duración de la estancia, la velocidad media, entre otros (Shoval y Isaacson, 2007). Así mismo, la información recogida mediante GPS permite cartografiar el uso social del espacio turístico e identificar los flujos de acceso, los flujos de agitación, las áreas de densidad y los espacios de descongestión. En definitiva, la principal ventaja de este tipo de análisis espacial es que proporciona una mayor y mejor comprensión de algunos de los comportamientos de los visitantes (Bauder, 2014) y permite poder incidir, de esta manera, en la gestión del espacio turístico y, en consecuencia, mejorar la calidad de la experiencia del turista.

Sin embargo, como apuntan Xiao-Ting y Bi-Hu (2012), los GPS no son absolutamente perfectos pues presentan algunas limitaciones a tener en cuenta. Así, en este sentido destaca en primer lugar, la pérdida de señal vía satélite, que aunque leve, es habitual. En segundo lugar, el comportamiento del visitante se puede ver alterado por el hecho de saber que está siendo monitorizado con el GPS. Finalmente, este método requiere una cierta inversión, si lo comparamos con otros métodos menos costosos, y necesita la complicidad de los visitantes para el estudio de campo.

Los estudios con GPS demuestran las tensiones propias de los flujos turísticos, tanto de acceso a los nodos principales, como de agitación en torno a los puntos de interés. La tendencia «ritual» de las dinámicas turísticas se reproduce en todos los ámbitos geográficos ya sean parques temáticos, espacios abiertos o ciudades metropolitanas, pero son mucho más evidentes en grandes áreas debido a que los procesos de «consumo» turístico acentúan la presión sobre unos pocos elementos del espacio turístico. Esta tensión justifica la necesidad de conocer las pautas del uso social del espacio por parte de los turistas, con el fin de ofrecer alternativas y herramientas de gestión eficaces tales como una señalización alternativa, la promoción de los nodos secundarios, la información diferenciada por clústeres, los itinerarios de disuasión o las medidas de capacidad de carga entre otras (Donaire y Galí, 2008).

Aunque la investigación turística a partir del uso de nuevas tecnologías (GPS, teléfonos móviles, tablets...) para el estudio del comportamiento turístico del espacio se encuentra en una fase inicial y, la mayoría de estudios en esta línea son sobre todo descriptivos y en espacios reducidos (Shoval et al., 2011), el número de trabajos cada vez es más cuantioso y diverso. Así, una de las áreas más comunes es la de los espacios naturales (Chhetri y 
Arrowsmith, 2008; Orellana et al., 2012; Wolf et al., 2012; Hallo et al., 2012). También empiezan a aparecer estudios en parques temáticos y espacios lúdicos (Zillinger, 2010; Russo et al., 2010; Pettersson y Zillinger, 2011; Birenboim et al., 2013); en grandes ciudades como Hong Kong (Shoval et al., 2011; Mckercher et al., 2012; Grinberger et al., 2014), Freiburg (Bauder, 2014) o Sydney y Camberra (Edward et al., 2010); y finalmente, en centros históricos y ciudades monumentales (Tchetchick et al., 2009; Van der Spek, 2010). Destacan en esta última línea, por ser los primeros, especialmente los trabajos de Shoval y Isaacsson (2006, 2007) en Heidelberg, en el centro histórico de Jerusalén, Nazaret, Acre y la vieja Jaffa. De forma paralela, se ha ampliado el área geográfica y se han registrado también datos de los movimientos de los visitantes en regiones turísticas, como las islas del Mar del Norte (Nielsen et al., 2010), Estonia (Ahas et al., 2007) o los valles alpinos italianos (Bruno et al., 2010).

Todos estos precedentes se han caracterizado por la selección de un ámbito geográfico que se corresponde con una determinada tipología turística ya fuera cultural, natural, metropolitana o temática. Ello permite identificar los mecanismos que intervienen en el comportamiento del turista ante un catálogo de recursos muy preciso tales como, los principales monumentos de las ciudades patrimoniales, las atracciones de un parque temático o, los nodos de una ciudad metropolitana. De todas formas, existen espacios turísticos que se caracterizan por la convivencia de recursos de naturaleza cultural, natural o recreativa, de manera que el visitante no solo debe escoger entre los diversos recursos del destino, sino también entre diversas categorías tipológicas. Este artículo explora el comportamiento de los visitantes en el Valle de Boí, un municipio del Pirineo catalán que se caracteriza por la presencia de recursos de naturaleza cultural, natural, y lúdica de alto valor. El objetivo es conocer de qué forma el visitante responde a un catálogo muy amplio de estímulos y hasta qué punto tiende a centrarse en una sola categoría o intenta abarcar el conjunto de la oferta del destino.

\section{METODOLOGÍA}

Mediante el análisis de los recorridos espacio-temporales realizados con el uso de GPS, nos interesa determinar la forma cómo los visitantes se comportan en el valle, tanto el movimiento espacial como el temporal. En este caso, el interés se centra en varios indicadores en particular, los tiempos de estancia, los tiempos de visita, las distancias recorridas, los lugares de interés, los nodos visitados y la intensidad de uso. Todos ellos ayudan a estimar la relación entre el visitante y el espacio visitado. Especialmente, nos interesa conocer la forma en la que el turista actúa en un medio caracterizado por la presencia simultánea de recursos que exigen pautas de uso del espacio muy diferentes.

\subsection{El objeto de estudio}

El Valle de Boí (mapa 1) es un municipio formado por nueve núcleos urbanos (Barruera, Boí, Cardet, Coll, Durro, Erill la Vall, Taüll, Pla de la Ermita y Sarais), situado en los Pirineos, en el norte de la provincia de Lérida. Está rodeado por un conjunto de cordilleras de más de 3.000 metros de altitud, que le otorgan un gran interés paisajístico. 


\section{Mapa 1 \\ MAPA DE SITUACIÓN DEL VALLE DE BOÍ}

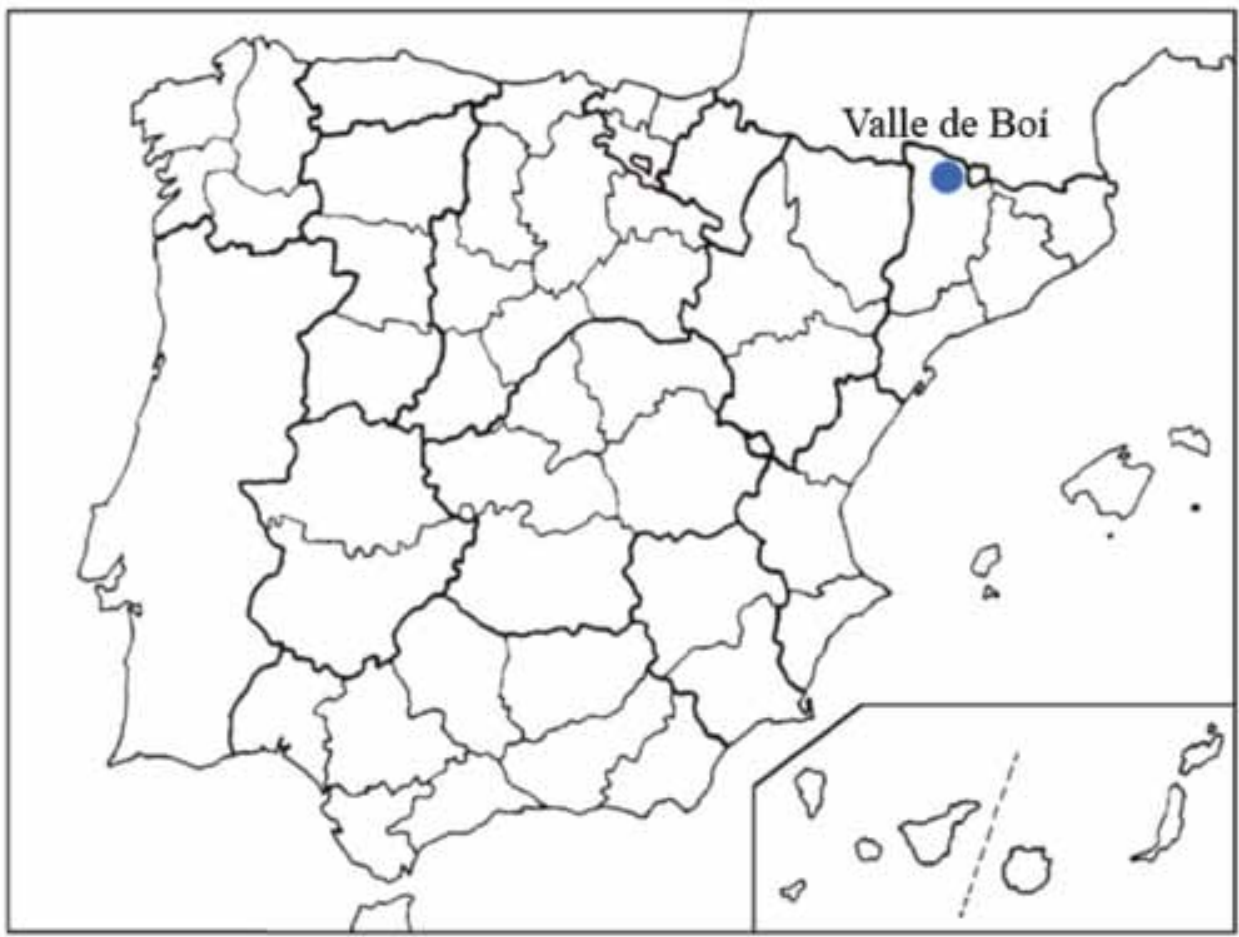

Cada núcleo urbano dispone de espacios patrimoniales de notable valor histórico y artístico tales como iglesias, ermitas, edificios civiles u otros elementos arquitectónicos y urbanos. De hecho, el conjunto de edificios románicos del valle, que comprende ocho iglesias románicas y una ermita, fue declarado Patrimonio de la Humanidad por la UNESCO en el año 2000 .

Además, el valle es uno de los puntos de entrada al Parque Nacional de Aigüestortes y Lago de San Mauricio, uno de los 14 parques nacionales de España y el único que hay en Cataluña. El parque constituye un espacio natural privilegiado y único en el sur de Europa.

Asimismo, el valle también cuenta con servicios turísticos de primer orden: una estación de esquí (Boí Taüll), con un dominio esquiable de 10.366 m.; y un Balneario, en Caldes de Boí, con la mayor variedad de aguas termales del mundo, por lo que ofrece una gran diversidad de tratamientos.

En definitiva, el Valle de Boí constituye un excelente laboratorio al aire libre, con todos los elementos que componen la experiencia turística: un extraordinario patrimonio cultural, unas características naturales excepcionales, servicios turísticos exclusivos y un rico patrimonio etnográfico característico de los valles pirenaicos. Por ello, los turistas encuentran en el valle muchos estímulos diferentes relativos al patrimonio, naturaleza o actividades lúdicas, lo que significa que deben seleccionar algunos elementos y rechazar otros. 


\subsection{El método: uso de los GPS}

La información para este estudio piloto fue recogida durante los meses de julio a octubre de 2012. Se recogieron 87 seguimientos realizados con GPS. De estos, se descartaron 8 por razones técnicas y problemas con la recepción vía satélite. La muestra definitiva fue, por tanto, de 79 seguimientos con GPS. La naturaleza exploratoria de este estudio se debe, no solo al interés de obtener algunos resultados preliminares, sino sobre todo al propósito de testar la metodología para poder corregir posibles problemas y mejorar la recuperación de datos de los dispositivos GPS. De aquí, el reducido tamaño de la muestra.

Los GPS se distribuyeron desde la Oficina de Turismo de Barruera, el primer núcleo del valle. Cada grupo de visitantes que podían ser un individuo, una pareja o un grupo familiar recibió solamente un dispositivo GPS, que devolvían en la misma OIT en un buzón previsto para ello en el momento que regresaban de su estancia en el valle. A cada visitante que formó parte de la muestra se le pidió, además de colgarse el GPS, responder un breve cuestionario con variables socio-demográficas.

El GPS, modelo QStarz Data Logger (BT-Q1000XT/TR-Q1000XT), registraba cada 10 segundos la posición del visitante midiendo las coordenadas de latitud y altitud con una precisión de pocos metros y en tiempo real. Los datos se guardaban automáticamente en el dispositivo y se descargaban en un PC (software QTravel) en el momento que el turista devolvía el GPS. Cada GPS tenía una autonomía de 72 horas. Los GPS se recargaban en la propia Oficina de Turismo durante el tiempo en que no estaban en uso.

El principal problema que surgió con los dispositivos de GPS, además de la pérdida de la señal del satélite, fue que las descargas se realizaban en formato $\mathrm{kml}$. El formato $\mathrm{kml}$ nos ha obligado a tener que hacer asociaciones y cálculos posteriores. De hecho, valoramos que hubiera sido preferible utilizar el fomato $s h p$ (ArcGIS), ya que la base de datos asociada es mucho más completa y el proceso metodológico más ágil y preciso. Así, con el formato $s h p$ cada registro de puntos del recorrido incluye la variable tiempo. Así mismo, permite acceder directamente a los archivos en ArcGIS, para el análisis geoespacial.

El tratamiento de la información espacial se basó en la delimitación de los polígonos que identifican cada recurso. En total, se han creado 23 polígonos, que se corresponden con los 23 recursos principales del valle. De esta forma, todos los registros del GPS situados en el interior del polígono son asignados al recurso con el que se ha vinculado. Ello permite conocer, por ejemplo, el tiempo destinado a visitar un núcleo de población o el Parque Nacional. Además, permite conocer la capacidad de atracción real de las iglesias románicas. Hasta la fecha, tan solo se disponía del recuento de personas que entraban a los templos mediante la compra de un ticket de entrada, sin conocimiento de sus perfiles. Con la ayuda del registro de los itinerarios, ahora puede conocerse el número de personas que se han interesado por las iglesias accediendo o sin acceder a ellas, cuando simplemente se contemplan desde el exterior, además de su perfil socio-demográfico.

\section{RESULTADOS Y DISCUSIÓN}

El principal resultado del análisis del uso social del espacio por parte de los turistas en el Valle de Boí es que los visitantes tienden a visitar un número muy elevado de 
recursos. El visitante optimiza al máximo su visita y crea un itinerario eficiente que le permite acceder a una suma de nodos y recursos muy amplia. De esta forma, el comportamiento en el valle es similar al que se ha identificado en espacios de una sola tipología turística, ya sea cultural, natural o lúdica. Por ejemplo, los estudios en las ciudades patrimoniales antes citados demuestran que los turistas acceden a un catálogo amplio de recursos del espacio y ésta es la tendencia que manifiestan también en un ámbito como el valle, que les ofrece recursos de naturaleza diversa. Esta suerte de zapping territorial es, por tanto, característica tanto de los espacios turísticos mono tipológicos como de los espacios poli tipológicos.

La tabla 1 muestra este comportamiento. Un turista medio visita varias localidades, el parque nacional y algunas iglesias románicas. Lógicamente, todos los turistas analizados han accedido al núcleo de Barruera, porque es el punto de recogida de los GPS. Pero además dos terceras partes han accedido al núcleo de Taüll o al Parque Nacional y tres cuartas partes han visitado el núcleo de Boí. Las iglesias de San Juan de Boí, San Félix de Barruera y San Clemente de Taüll, el icono del valle, se mueven en torno al $50 \%$ del total de visitantes. Así mismo, el porcentaje del resto de iglesias y de núcleos de población no es baladí.

Tabla 1

PORCENTAJE DE VISITANTES EN CADA RECURSO

\begin{tabular}{|l|r|}
\hline Espacio visitado & Porcentaje visitas (\%) \\
\hline Barruera & 100 \\
\hline Boí & 78 \\
\hline Taüll & 65 \\
\hline PN Lago San Mauricio & 61 \\
\hline San Juan de Boí & 61 \\
\hline San Félix de Barruera & 55 \\
\hline San Clemente de Taüll & 48 \\
\hline Erill la Vall & 41 \\
\hline Durro & 32 \\
\hline Santa María de Taüll & 32 \\
\hline PN Presa & 30 \\
\hline Balneario & 29 \\
\hline Caldes de Montbuí & 28 \\
\hline Santa Eulalia de Erill la Vall & 27 \\
\hline La Natividad de Durro & 20 \\
\hline La ermita de San Quirc de Durro & 19 \\
\hline Santa María de Cardet & 16 \\
\hline Resort & 8 \\
\hline Cardet & 3 \\
\hline
\end{tabular}

La prueba piloto revela también que los visitantes del Valle de Boí permanecen en el valle una media de 20 horas, con una desviación estándar de 7 horas; estos descriptivos 
nos muestran que el visitante del Valle responde tanto a la tipología de excursionista como a la de turista en destinos rurales-culturales (Royo, 2009). En ambos casos, una estancia breve y un elevado número de nodos visitados dibujan un movilidad interna muy elevada y, al mismo tiempo, un consumo efímero de cada elemento. Así, aunque con una elevada desviación de $57 \mathrm{Km}$, un turista medio recorre $98 \mathrm{Km}$, lo que supone una velocidad media de $5 \mathrm{~km} / \mathrm{h}$. Si tenemos en cuenta que una parte importante de la estancia es estática debida a pernoctaciones, contemplaciones o restauraciones, vemos que el consumo turístico del valle se caracteriza por una extrema movilidad. Los flujos de agitación, esto es, los recorridos internos en el valle, son muy frecuentes y una de las características más relevantes del comportamiento del visitante. Hay que tener en cuenta que un $30 \%$ de la muestra se ha desplazado fuera del valle durante el período de registro, normalmente a espacios colindantes como el Valle de Arán. Por eso, el mapa sobre los elementos visitados del valle muestra una ocupación muy amplia del conjunto del territorio.

Un número elevado de elementos visitados en poco tiempo exige que el tiempo medio de visita sea muy breve. La tabla 2 muestra el número de minutos dedicados a la visita de cada iglesia. Como se puede comprobar la media es muy reducida, incluso con el factor de corrección de las desviaciones. Es decir, parece ser que los nodos son más observados que visitados. En definitiva, el patrón de comportamiento del visitante consiste en reconocer unos determinados nodos socialmente asentados en el imaginario turístico. Es decir, los turistas se comportan en el valle siguiendo unas pautas de consumo pre-establecidas.

Los espacios donde el visitante dedica más tiempo a la observación o contemplación son las iglesias de Boí y Taüll. Especialmente, San Clemente de Taüll con una media de 9 minutos y 6 segundos, que es un tiempo sensiblemente superior al tiempo medio de visita del resto de nodos. El factor que explica este incremento de tiempo es la pintura mural de San Clemente ubicada en el ábside central de la iglesia, icono del arte románico catalán, y considerada uno de los ejemplos más puros del románico europeo ${ }^{1}$.

Tabla 2

TIEMPO (EN MINUTOS) DE VISITA EN CADA IGLESIA

\begin{tabular}{|l|c|c|c|}
\hline \multicolumn{1}{|c|}{ Iglesia } & Media & Desviación & Máx. \\
\hline San Clemente de Taüll & 9,6 & 8,9 & 32,3 \\
\hline Santa María de Taüll & 2,1 & 2,1 & 7,5 \\
\hline San Juan de Boí & 5,8 & 3,6 & 13,7 \\
\hline Santa Eulalia de Erill & 6,4 & 6,4 & 20,3 \\
\hline San Félix de Barruera & 2,6 & 1,8 & 6,0 \\
\hline La Natividad de Durro & - & - & - \\
\hline San Quirc de Durro & - & - & - \\
\hline Santa María de Cardet & 3,3 & 1,5 & 5,8 \\
\hline
\end{tabular}

1 El mural original se encuentra en el Museo Nacional de Arte de Cataluña (MNAC) en Barcelona. 
Podemos hablar, en general, de la identificación de un comportamiento que denominamos fast look, es decir, una visita rápida y superficial del máximo posible de nodos en el mínimo tiempo posible. Los turistas no tienen una relación profunda con los elementos visitados, sino que se limitan a coleccionar piezas del mosaico turístico y donde la fotografía ayuda a fijar y complementar los recuerdos de una experiencia superficial.

Otro aspecto que se ha considerado en el análisis de los registros con los GPS ha sido el tiempo de visita en el interior de las iglesias versus el tiempo de visita del exterior. El resultado, pone de manifiesto que son nodos de exterior. Es decir, en la mayoría de iglesias los visitantes han dedicado más tiempo a la visita del exterior que a la visita interior, con tres excepciones. En primer lugar, San Clemente de Taüll, la iglesia con mayor número de piezas artísticas en el interior tales como las imágenes del altar, de la pila bautismal, o de la mandorla con el Pantocrátor. En segundo lugar, la iglesia de Santa Eulalia de Erill la Vall debido a que desde el interior de la misma se puede acceder al magnífico campanario románico de seis plantas, lo que incrementa notablemente el tiempo de estancia en el interior. La tercera excepción, Santa María de Cardet, una pequeñísima iglesia ubicada en un enclave donde la visita exterior solo permite la vista de la fachada principal, en cambio la visita interior permite acceder a la zona del coro en el piso superior, además de la nave basilical en la planta principal.

Así pues, otra característica del comportamiento de los turistas en el Valle de Boí es que se basa esencialmente en el consumo del exterior de los nodos o de atributos del espacio que son fácilmente visibles. La visita, tal y como ya se ha comentado antes, responde más al patrón comportamental de un ritual rápido con breve visión general y alguna fotografía que a un esfuerzo de entender y sentir el valor del patrimonio.

No obstante, la organización de las piezas que integran la oferta turística del valle es muy jerárquica. Este principio ya ha sido detectado en todos los estudios sobre comportamiento de los visitantes en destinos tanto culturales como naturales o lúdicos. El sistema turístico tiende a organizar todo el catálogo de oportunidades de visita de forma muy vertical. Unos pocos elementos son universales y forman parte del conjunto de los recorridos; y a medida que se amplía el tiempo de visita, se accede a otros niveles inferiores de la jerarquía turística. Por tanto, el comportamiento de los visitantes es relativamente ritual y tiende a reproducir unas pautas de consumo muy previsibles, que generan tensiones en el destino tales como la saturación de las áreas centrales, la ausencia de turistas en los espacios periféricos o los comportamientos homogéneos tanto espaciales como temporales.

El Valle de Boí no es una excepción. Si nos centramos, por ejemplo en la visita a las iglesias que son patrimonio de la humanidad constatamos que solamente unas pocas acaparan la mayor parte de los registros. Así, tal y como muestra la figura 1, el 18,8\% de los visitantes entran en una única iglesia del conjunto de iglesias patrimonio de la humanidad del valle. El 41,3\% visitan entre dos y tres iglesias, el 28,9\% cuatro o más iglesias, y solo el 11,3\% de los visitantes, aquellos interesados básicamente en la naturaleza, no ha visitado ninguna iglesia. La media de iglesias visitadas es de 2,73 y se dedica un tiempo medio de 12 minutos. Los resultados evidencian la importancia de las iglesias románicas de Boí y Taüll como el gran atractivo del valle. Son nodos con una gran capacidad de atracción y que consiguen captar el interés del visitante. Por ello, el comportamiento turístico del Valle de Boí responde al modelo de territorio nodal o de sights. 


\section{NÚMERO DE IGLESIAS VISITADAS (\%)}

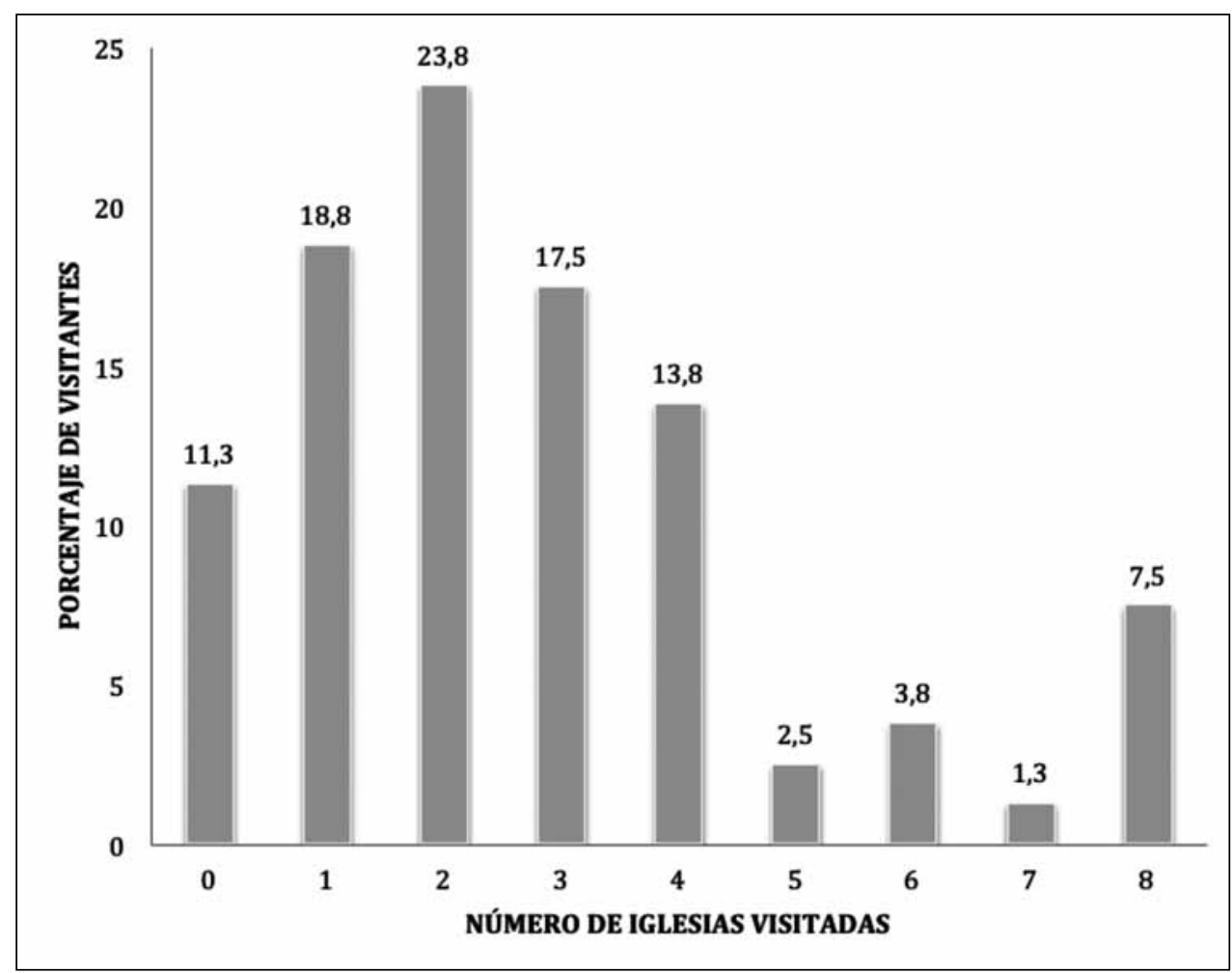

\section{CONCLUSIONES}

El comportamiento turístico ha sido objeto de estudio académico desde hace tiempo. Este ha tomado como base de sus análisis diferentes métodos de recopilación de datos a lo largo de las décadas. Desde los comunes diarios de viajes y mapas de comportamiento, hasta la observación participada y no participada, pasando por las encuestas y paneles. Últimamente, los Sistemas de Posicionamiento Global (GPS) se han empezado a utilizar para registrar el movimiento espacio-temporal de los turistas.

Con el objetivo de testar la metodología de obtención de datos utilizando GPS, se eligió el Valle de Boí, un espacio que combina naturaleza, cultura y oferta lúdica a la vez, con el objetivo de identificar algunos de los comportamientos espacio-temporales de los turistas del valle y definir la relación del visitante con el espacio visitado.

Los resultados preliminares de este estudio, de carácter exploratorio, muestran que es posible extender el uso de GPS a espacios abiertos, con una oferta de recursos amplia, y un intervalo temporal dilatado. Aunque el reducido número de casos no permite extraer conclusiones estadísticamente representativas, sí podemos constatar que los primeros 
resultados nos han permitido estudiar el uso de las diferentes piezas del sistema. La secuencia espacio-temporal de la visita y el grado de intensidad de uso de cada elemento.

De forma preliminar el estudio pone de manifiesto que los turistas intentan visitan y el máximo número posible de elementos, independientemente de su naturaleza cultural, lúdica o natural. En segundo lugar, este consumo es extremadamente efímero, muy fugaz. Los visitantes recorren un itinerario muy amplio en un lapso temporal muy breve, con lo que el tiempo medio de estancia en cada nodo es muy bajo. Podemos hablar de fast look, denominación que damos a un comportamiento del visitante caracterizado por una mirada rápida y superficial al elemento patrimonial. Finalmente, hemos detectado que los elementos se organizan de forma jerárquica, de manera que el comportamiento de los visitantes se homogeneiza al seguir tal jerarquía de interés.

Como principal limitación del estudio cabe decir que a pesar de las ventajas del uso de estas tecnologías, todavía hay aspectos del comportamiento de los visitantes tales como la mirada turística, el interés por determinados elementos, la percepción del destino, su imagen y formación o el nivel de satisfacción que no pueden ser analizados con el uso de los GPS. Estos aspectos precisan de técnicas como la observación directa participada o no participada (Hartmann,1988; Keul y Küheberger,1997; Galí y Donaire, 2006, 2010), los diarios espacio-temporales (Thorthon et al., 1997), el análisis de las fotografías realizadas por los turistas (Mackay y Couldway, 2004; Garrod, 2008; Donaire y Galí, 2011; Stylianou-Lambert, 2012), o los habituales cuestionarios post-visita o entrevistas cualitativas (Royo, 2009). Las investigaciones futuras deberían tratar de complementar los resultados obtenidos con los GPS con los obtenidos a través de estas otras metodologías.

\section{BIBLIOGRAFÍA}

AHAS, R., AASA, A., MARK, U., PAE, T. y KULL, A. (2007): «Seasonal tourism spaces in Estonia: Case study with mobile positioning data», Tourism Management, 28(3), pp. 898-910.

BAUDER, M. (2014): «Using GPS Supported Speed Analysis to Determine Saptial Visitor Behaviour», International Journal of Tourism Research. Doi: 10.1002/jtr.1991

BIRENBOIM, A., ANTÓN, S., RUSSO, A.P. y SHOVAL, N. (2013): «Temporal Activity Patterns of Theme Park Visitors», Tourism Geographies, 15(4), pp. 601-619.

BOWEN, D. (2002): «Research through Participant Observation in Tourism: A Creative Solution to the Measurement of Consumer Satisfaction/Dissatisfaction (CS/D) among tourists», Journal of Travel Research, 41(4), pp. 4-14.

BRUNO, A., GASCA, E., MAURO, S., POLliCHINO, G., SACERDOTTI, S.L. y STUPINO,F. (2010): «Undersatnding tourist behaviour in wide áreas using GPS technologies». E-Review of Tourism Reseach (Special Section: ENTER 2010 Information and comuniation technologies in tourism. Short Paper Track, 424). Vienna: SpringerVerlag.

CATON, K., y SANTOS C.A. (2008): «Closing the hermeneutic circle? Photographic Encounters with the Others», Annals of Tourism Research, 35(1), pp. 7-26.

CHHETRI, P. y ARROWSMITH, C. (2008): «GIS-based Modelling Recreational Potential of Nature-Based Tourist Destinations», Tourism Geographies, 10(2), pp. 233-257. 
CRANDALL, D., BACKSTORM, L., HUTTENLOCHER, D., y KLEINBERG J. (2009): «Mapping the World's Photos». In Proceedings of the 18th International WWW Conference (pp. 761-770). New York: Association for Computing Machinery (ACM).

DEBBAGE, K.G. (1991): «Spatial behaviour in a bahamian resort», Annals of Tourism Research, 18(2), pp. 251-268.

DONAIRE, J.A., CAMPRUBÍ, R. y GALÍ, N. (2014): «Tourists clusters from Flickr travel photography», Tourism Management Perspectives, 11, pp. 26-33.

DONAIRE, J.A. y GALÍ, N. (2011): «La imagen turística de Barcelona en la comunidad Flickr», Cuadernos de Turismo, 27, pp. 291-303.

DONAIRE, J.A. y GALÍ, N. (2008): «Modeling tourist itineraries in heritage cities. Routes around the Old District of Girona», Pasos. Revista de Turismo y Patrimonio Cultural, 6(3), pp. 435-449.

GALÍ, N. y DONAIRE, J.A. (2006): «Visitor's behavior in Heritage Cities: The Case of Girona», Journal of Travel Research, 44(4), pp. 442-448.

GALÍ, N. y DONAIRE, J.A. (2010): «Direct Observation as a methodology for effectively defining tourist behaviour». E-Review of Tourism Reseach (Special Section: ENTER 2010 Information and comunication technologies in tourism. Short Paper Track, 424). Vienna: Springer-Verlag.

GARROD, B. (2008): «Exploring place perception. A Photo-based Analysis», Annals of Tourism Research, 35(2), pp. 381-401.

GRINBERGER, A.Y., SHOVAL, N. y MCKERCHER, B. (2014): «Typologies of tourists' time-space consumption: A new approach using GPS data and GIS tools», Tourism Geographies, DOI:10.1080/14616688.2013.869249

HALDRUP, M. (2004): «Laid-Back Mobilities: Second-Home Holidays in Time and Space», Tourism Geographies, 6(4), pp. 434-454.

HALLO, J., BEECO, J.A., GOETCHEUS, C., MCGEE, J., GARD, N. y NORMAN, W. (2012): «GPS as a Method for Assessing Spatial and Temporal Use Distributions of Nature-Based tourists», Journal of Travel Research, 51(5), pp. 591-606.

HÄGERSTRAND, T. (1970): «What about People in Regional Science?», Regional Science Association Papers, 24, pp. 7-21.

HARTMANN, R. (1988): «Combining Field Methods in Tourism Research», Annals of Tourism Research, 5(1), pp. 88-105.

HAYWOOD, K.M. (1990): «Visitor-employed photography: an urban visit assessment», Journal of Travel Research, 29(1), pp. 25-29.

LEW, A. y MCKERCHER, B. (2006): «Modeling tourist movements. A Local Destination Analysis», Annals of Tourism Research, 33(2), pp. 403-423.

KEUL A. y KÜHEBERGER, A. (1997): «Tracking the Salzburg Tourist», Annals of Tourism Research, 24(4), pp. 1.008-1.012.

MACKAY, K.J., y COULDWAY, C.M. (2004): «Using Visitor-Employed Photography to Investigate Destination Image», Journal of Travel Research, 42(4), pp. 390-396.

MCKERCHER, B., SHOVAL, N., NG, E. y BIRENBOIM, A. (2012): «First y Repeat Visitor Behaviour: GPS Tracking and GIS Analysis in Hong Kong», Tourism Geographies, 14(1), pp. 147-161. 
MOLTZ, JG. (2010): «Performing Global Geographies: Time, Space, Place and Pace in Narratives of Round-the-World Travel», Tourism Geographies, 12(3), pp. 329-348.

MURPHY, E. (1992): «Data gathering for community-oriented tourism planning: case study of Vancouver Island, British Columbia», Leisure Studies, 11(1), pp.65-79.

NIELSEN, N.; HARDER, H.; TRADISAUKAS, N. y BLICHFELDT, B.S. (2010): «Approaches to GPS-survey of tourist movements within a North Sea island destination». E-Review of Tourism Reseach (Special Section: ENTER 2010 Information and comunication technologies in tourism. Short Paper Track, 424). Vienna: Springer-Verlag.

ORELlANA, D., BREGT, A. K., LIGTENBERG, A. y WACHOWICZ, M. (2012): «Exploring visitor moviments patterns in natural recreational areas». Tourism Management, 33(3), pp. 672-682.

PEARCE, D. (1988): «Tourist Time-Budget», Annals of Tourism Research, 15(1), pp. 106-121.

PETTERSSON, R. y ZILLINGER, M. (2011): «Time and Space in Event Behaviour: Tracking Visitors by GPS», Tourism Geographies, 13(1), pp. 1-20.

ROYO-VELA, M. (2009): «Rural-cultural excursion conceptualization: A local tourism marketing management model based on tourist destination image measurement», Tourism Management, 30(3), pp. 419-428.

RUSSO, A.P., ANTON, S., y SHOVAL, N. (2010): «Advanced visitor tracking analysis in practice: Explorations in the Port Aventura Theme park and insights for a future research agenda». In U. Gretzel, R. Law, y M. Fuchs (Eds.), Information and comunication technologies in tourism 2010. Proceedings of the international conference ENTER 2010 (pp. 159-170).Vienna: Springer-Verlag.

SHOVAL, N., MCKERCHER, B., NG, E. y BIRENBOIM, A. (2011): «Hotel Location and Tourist activity in cities», Annals of Tourism Research, 38(4), pp. 1594-1612.

SHOVAL, N. y ISAACSON, M. (2006): «Application of Tracking Tecnologies to the Study of Pedestrian Spatial Behavior», The Professional Geographer, 58(2), pp. 172-183.

SHOVAL, N. y ISAACSON, M. (2007): «Tracking Tourisnt in The Digital Age», Annals of Tourism Research, 34(1), pp. 141-159.

STYLIANOU-LAMBERT, T. (2012): «Tourists with Cameras: Reproducing or Producing», Annals of Tourism Research, 39(4), pp. 1817-1838.

TCHETCHICK, A., FLEISCHER, A. y SHOVAL, N. (2009): «Segmentation of Visitors to a Heritage Site Using High-resolution Time-space Data», Journal of Travel Research, 48(2), pp. 216-229.

THORNTON, P.R.; WILLIAMS A.M. y SHAW, G. (1997): «Revisiting time-space diaries: an exploratory case study of tourist behaviour in Cornwll, England», Environment and Planning, 29, pp. 1.847-1.867.

VAN DER SPEK, S.C. (2010): «Tracking tourists in historic city centres». In U. Gretzel, R. Law, y M. Fuchs (Eds.). Information and comunication technologies in tourism 2010. Proceedings of the International conference ENTER 2010 (pp. 185-196). Vienna: Springer-Verlag.

WOLF, I.D., HAGENLOH, G. y CROFT, D.B. (2012): «Visitors monitoring along roads and hiking trails: How to determine usage levels in tourist sites», Tourism Management, 33, pp. 16-28. 
XIAO-TING, H. y BI-HU, W. (2012): «Intra-attraction Tourist Spatial-Temporal Behaviour Patterns», Tourism Geographies, 14(4), pp. 625-645.

ZILLINGER, M. (2010): «Experience Tracking-Evaluating Methods for Studying Experiences in Time and Space». E-Review of Tourism Reseach (Special Section: ENTER 2010 Information and comunication technologies in tourism. Short Paper Track, 424). Vienna: Springer-Verlag. 
